\title{
Ipilimumab/Nivolumab Regimen
}

National Cancer Institute

\section{Source}

National Cancer Institute. Ipilimumab/Nivolumab Regimen. NCI Thesaurus. Code

C154272.

A regimen consisting of ipilimumab and nivolumab that can be used for the treatment of kidney cancer, colorectal cancer and melanoma. 